\title{
MOMENTS ON RIEMANN SURFACES AND HYPERELLIPTIC ABELIAN INTEGRALS
}

\author{
L. GAVRILOV AND F. PAKOVICH
}

\begin{abstract}
In the present paper we solve the following different but interrelated problems: (a) the moment problem on Riemann surfaces, (b) the vanishing problem of polynomial Abelian integrals of dimension zero on the projective plane, (c) the vanishing problem of polynomial hyperelliptic Abelian integrals.
\end{abstract}

\section{Contents}

1. Introduction

2. Moments on Riemann surfaces and zero-dimensional Abelian integrals 3

2.1. Solution of the moment problem in the case of generic position $\quad 7$

3. Vanishing of genus zero Abelian integrals 9

3.1. Description of the irreducible $G_{P}$-invariant subspaces of $\mathbb{Q}^{n}$. $\quad 9$

$\begin{array}{lll}3.2 \text {. Decomposition of } V_{\delta} \text { into a direct sum of irreducible subspaces } & 10\end{array}$

3.3. Description of the spaces $Z_{U_{d}}$. 11

3.4. Corollaries 14

3.5. Polynomial moment problem on a system of intervals with weights 15

4. Vanishing of hyperelliptic Abelian integrals $\quad 17$

4.1. Vanishing and the moment problem. 17

4.2. The Cauchy integral related to $I \quad 18$

4.3. Hyperelliptic Abelian integrals along ovals. 23

References $\quad 25$

\section{Introduction}

In the present paper we solve the following different but interrelated problems:

(a): Let $f$ and $\omega$ be a function and a one-form meromorphic on a compact Riemann surface $R$ and $\gamma \subset R$ be a closed curve. In Section 2 we give necessary and sufficient conditions for all the "moments"

$$
m_{s}=\int_{\gamma} f^{s} \omega, \quad s \geq 0
$$

to vanish identically. These condition are expressed in terms of the vanishing of a finite collection of algebraic functions, which can be interpreted as Abelian integrals of dimension zero on $R$.

Date: November 21, 2018.

Part of the paper was written while the first author was visiting the Ben-Gurion University. He is obliged for the hospitality. 
(b): Motivated by problem (a), we describe in Section 3 necessary and sufficient conditions for the identical vanishing of polynomial Abelian integrals of dimension zero on the projective plane.

(c): Finally, in Section 4 we apply the results obtained to the problem of the identical vanishing of hyperelliptic Abelian integrals of the form

$$
I(t)=\int_{\gamma(t)} P(x, y) d x+Q(x, y) d y, \quad P, Q \in \mathbb{C}[x, y]
$$

where $\gamma(t) \in H_{1}\left(\Gamma_{t}, \mathbb{Z}\right)$ is a continuous family of 1-cycles and

$$
\Gamma_{t}=\left\{(x, y) \in \mathbb{C}^{2}: y^{2}-f(x)=t\right\}, \quad f \in \mathbb{C}[x]
$$

is a family of hyperelliptic curves.

The moment vanishing problem (a) has been studied by several authors in the last years, in relation with the center problem for the Abel equation

$$
\frac{d y}{d z}=p(z) y^{2}+q(z) y^{3}, \quad p, q \in \mathbb{C}[z],
$$

see [2] for an extensive list of references. The solution that we present here in terms of zero-dimensional Abelian integrals is inspired by the approach of [13], [15].

Zero-dimensional Abelian integrals were introduced recently in [6], in an attempt to verify certain conjectures concerning the 16th Hilbert problem in dimension zero. In particular, the problem of identical vanishing of such zero-dimensional integrals on the Riemann sphere for simple cycles has been studied and solved in $[6,3]$. Notice however that in this case the problem reduces to the finding of conditions implying that for a pair of polynomials $f, g$ the equality $g\left(f_{i}^{-1}(z)\right) \equiv g\left(f_{j}^{-1}(z)\right)$ holds for two different branches of the function inverse to $f$, and in such a form the problem was studied and solved earlier (see e.g. [16], [10]). In the general case the problem (b) is to find conditions implying the equality

$$
\sum_{i=1}^{n} a_{i} g\left(f_{i}^{-1}(z)\right) \equiv 0, \quad n=\operatorname{deg} f
$$

for arbitrary given rational $a_{i} \in \mathbb{Q}$. Essentially, in an implicit form a solution of this problem was already done in [14] as an ingredient of the proof of the so called polynomial moment problem. However, having in mind possible applications, for the reader convenience we present here a detailed and full exposition which is selfcontained up to a single purely algebraic result of [14]. Notice that a partial solution of problem (b), also basing on results of [14], has been recently done in [1].

The last problem (c) we solve concerns the identical vanishing of complete hyperelliptic Abelian integrals of the form (2). Although this problem is of independent interest, we are once again motivated by applications to the 16th Hilbert problem. Namely, it is well known that if a limit cycle of the perturbed plane foliation

$$
d\left(y^{2}-f(x)\right)+\varepsilon(P(x, y) d x+Q(x, y) d y)=0, \quad \varepsilon \sim 0
$$

bifurcates from the period orbit $\gamma\left(t_{0}\right) \subset \Gamma_{t_{0}}$ of the non-perturbed foliation, then the Abelian integral $I(t)$ defined by (2) vanishes at $t_{0}$. This is a corollary of the representation

$$
P_{\varepsilon}(t)=t+\varepsilon I(t)+o(\varepsilon)
$$

of the first return map $P_{\varepsilon}$ associated to the family of periodic orbits $\gamma(t)$. The situation in which $I(t) \equiv 0$ is exceptional, and this phenomenon is related to the 
singularities of the algebraic set of plane integrable foliation. Notice that even for plane polynomial vector fields of degree three, the structure of the set of integrable foliations is unknown [9] (for quadratic vector fields it is due to Dulac (1908) and Kapteyn (1912)). On the other hand, the identical vanishing of $I(t)$ only shows that the foliation (3) is integrable "at a first order", and the study of the higher order terms in the expansion (4) is needed in order to solve the associated center problem on the plane [5, 7].

The key idea to solve problem (c) is to interpret the derivatives of $I(t)$ as moments (1) for a certain choice of $R, f$ and $\omega$. Then the identical vanishing of $I(t)$ turns out equivalent, according to (a), to the identical vanishing of a collection of Abelian integrals of dimension zero. Furthermore, these Abelian integrals essentially reduce to the ones studied in (b).

\section{Moments on Riemann surfaces and zero-dimensional Abelian INTEGRALS}

Let $f, \omega$ be respectively a function and a one-form meromorphic on a compact Riemann surface $R$, and let $\gamma \subset R$ be a closed rectifiable curve which avoids the poles of $f$. Then the moments (1) are well defined. In this section we shall suppose, for simplicity, that the set of poles of $\omega$ is contained in the set of poles of $f$. We shall give necessary and sufficient conditions for the generating function

$$
J(t)=J(\omega, f, \gamma, t)=-\sum_{i=0}^{\infty} \frac{m_{i}}{t^{i+1}}=\int_{\gamma} \frac{\omega}{f-t}, \quad t \sim \infty
$$

of the moments $m_{i}$ to vanish identically or, more generally, to be rational. For this purpose we follow closely [15], where the genus zero case, $R=\mathbb{C P}^{1}$, was studied in details.

Consider the induced map

$$
\begin{aligned}
f: R & \rightarrow \mathbb{C P}^{1} \\
x & \mapsto[f(x): 1]
\end{aligned}
$$

and let $\left\{c_{1}, c_{2}, \ldots c_{k}\right\}$ be the set of all finite critical values of $f$. For a regular generic value $c_{0} \in \mathbb{C}$, consider the "star" $S \subset \mathbb{C}$ consisting of the segments $\left[c_{0}, c_{i}\right]$, $i=1,2 \ldots k$. It is not hard to show, using the assumption that $S$ contains all finite critical values of $f$, that one can continuously deform the path $\gamma$, without changing the corresponding function $J(t)$, in such a way that the image $f(\gamma)$ will be contained in $S$ (the explicit construction is given below). Therefore (1) becomes

$$
m_{s}=\int_{\gamma} f^{s} \omega=\int_{\gamma} f^{s} \frac{\omega}{d f} d f=\sum_{i=1}^{k} \int_{c_{0}}^{c_{i}} \varphi_{i}(z) z^{s} d z
$$

where $\varphi_{i}$ is an appropriate sum of branches of the algebraic function

$$
\frac{\omega}{d f} \circ f^{-1}
$$

in some simply-connected domain $U$ containing $S \backslash\left\{c_{1}, c_{2}, \ldots c_{k}\right\}$.

Clearly,

$$
J(t)=\sum_{i=1}^{k} J_{i}(t), \text { where } J_{i}(t)=\int_{c_{0}}^{c_{i}} \frac{\varphi_{i}(z)}{z-t} d z .
$$


Further, the functions $J_{i}(t)$ and therefore $J(t)$ allow for an analytic continuation on $\mathbb{C P}^{1} \backslash\left\{c_{1}, c_{2}, \ldots c_{k}\right\}$. On the other hand, by a well-known property of Cauchy type integrals, the limits of the function $J(t)$ when $t$ approaches to a point $t \in\left[c_{0}, c_{i}\right]$ from the "left" and "right" sides of $\left[c_{0}, c_{i}\right]$ are related by the equality

$$
J_{i}^{+}(t)-J_{i}^{-}(t)=2 \pi \sqrt{-1} \varphi_{i}(t) .
$$

Therefore, if the generating function $J(t)$ vanishes identically (or just allows for a single-valued analytical continuation), then the algebraic functions $\varphi_{i}, i=1,2 \ldots k$ defined by (7) vanish identically. Of course, $\varphi_{i}=0$ also implies that $J=0$.

The study of conditions implying the vanishing of the algebraic functions $\varphi_{i}$ is a priori a simpler problem than the initial one. Furthermore, the functions $\varphi_{i}$ allow for a remarkable interpretation as 0-dimensional Abelian integrals which we describe now.

Consider the singular fibration (6) with fibers

$$
f^{-1}(z)=\left\{f_{1}^{-1}(z), f_{2}^{-1}(z), \ldots, f_{d}^{-1}(z)\right\}
$$

where $d$ is the degree of $f$. For $z \neq c_{i}, \infty$ define the (reduced) zero-homology group

$$
\widetilde{H}_{0}\left(f^{-1}(z), \mathbb{Z}\right)=\left\{n_{1} f_{1}^{-1}(z)+n_{2} f_{1}^{-1}(z)+\ldots n_{d} f_{d}^{-1}(z): \sum n_{i}=0, n_{i} \in \mathbb{Z}\right\} .
$$

It is a free $\mathbb{Z}$-module generated by

$$
f_{1}^{-1}(z)-f_{d}^{-1}(z), f_{2}^{-1}(z)-f_{d}^{-1}(z), \ldots, f_{d-1}^{-1}(z)-f_{d}^{-1}(z)
$$

and its dual space is denoted by $\widetilde{H}^{0}\left(f^{-1}(z), \mathbb{C}\right)$. The map (6) induces homology and co-homology bundles with the base $\mathbb{C} \backslash\left\{c_{1}, \ldots, c_{k}\right\}$ and fibers $\widetilde{H}_{0}\left(f^{-1}(z), \mathbb{Z}\right)$ and $\widetilde{H}^{0}\left(f^{-1}(z), \mathbb{C}\right)$. The continuous families of cycles

$$
f_{i}^{-1}(z)-f_{j}^{-1}(z) \in \widetilde{H}_{0}\left(f^{-1}(z), \mathbb{Z}\right)
$$

generate a basis of locally constant sections of a canonical connection on the homology bundle (the Gauss-Manin connection). Clearly a meromorphic function $g$ on $R$ defines a meromorphic section of the co-homology bundle, and we may define a zero-dimensional Abelian integral as follows (see [6]).

Definition 2.1. A zero-dimensional Abelian integral is an algebraic function

$$
\int_{\delta(z)} g=n_{1} g\left(f_{1}^{-1}(z)\right)+n_{2} g\left(f_{1}^{-1}(z)\right)+\cdots+n_{d} g\left(f_{d}^{-1}(z)\right),
$$

where $g$ is a meromorphic function on $R$ and

$$
\delta(z)=n_{1} f_{1}^{-1}(z)+n_{2} f_{2}^{-1}(z)+\ldots n_{d} f_{d}^{-1}(z) \in \widetilde{H}_{0}\left(f^{-1}(z), \mathbb{Z}\right)
$$

is a continuous family of 0-cycles.

Clearly, the functions $\varphi_{i}$ in (8) may be interpreted as zero-dimensional Abelian integrals

$$
\varphi_{i}(z)=\int_{\delta_{i}(z)} \frac{\omega}{d f},
$$

where

$$
\delta_{i}(z)=\sum_{j=1}^{d} n_{i j} f_{j}^{-1}(z)
$$

and $n_{i j}$ are suitable integers (to be computed bellow). 
To resume, we proved that if the set of poles of $\omega$ is contained in the set of poles of $f$, then the following statement is true.

Theorem 2.1. The moments

$$
m_{s}=\int_{\gamma} f^{s} \omega, \quad s \geq 0
$$

vanish if and only if the zero-dimensional Abelian integrals

$$
\varphi_{i}(z)=\int_{\delta_{i}(z)} \frac{\omega}{d f}, \quad i=1,2, \ldots, k
$$

vanish.

Of course, to apply the Theorem 2.1 we need the precise values of the integer numbers $n_{i j}$ defined by (13). Following [15], we may compute these numbers as follows. Consider the pre-image of the star $S$ under $f$

$$
\lambda_{f}=f^{-1}(S) \subset R
$$

as a graph embedded in the Riemann surface $R$. This graph, called a constellation, in a sense is a "combinatorial portrait" of the corresponding covering (see [8] for details and different versions of this construction). We will show that the combinatorial properties of $\lambda_{f}$ determine $n_{i j}$.

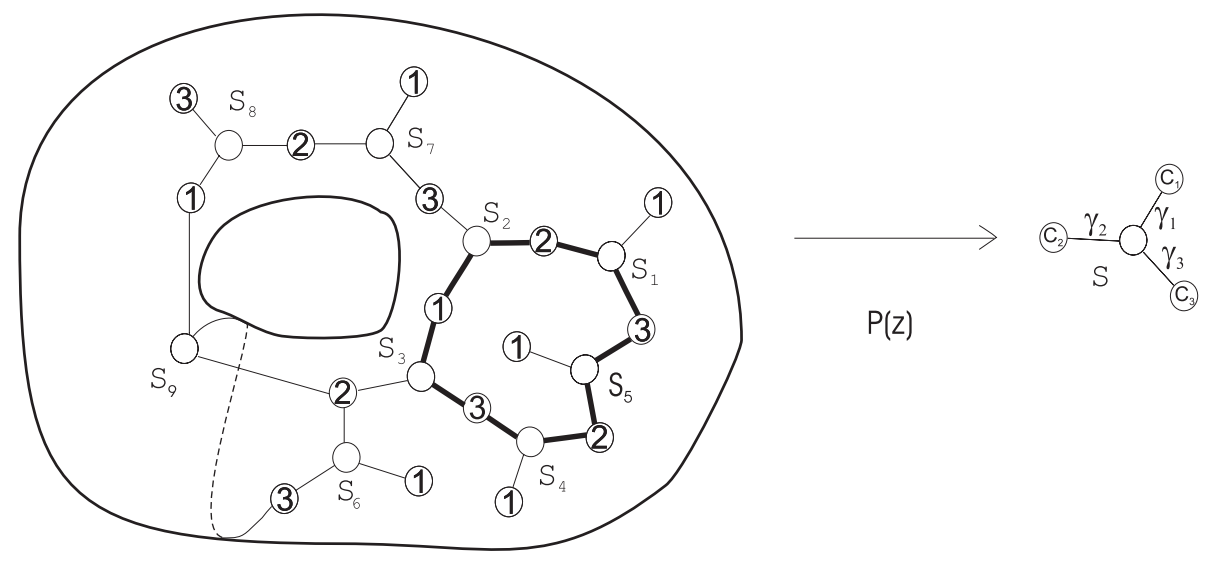

FiguRE 1

By construction, the restriction of $f(z)$ on $R \backslash \lambda_{f}$ is a covering of the topological punctured disk $\mathbb{C P}^{1} \backslash\{S \cup \infty\}$ and therefore $R \backslash \lambda_{f}$ is a disjoint union of disks. This implies that the graph $\lambda_{f}$ is connected and the faces of $\lambda_{f}$ are in a one-to-one correspondence with poles of $f(z)$. For each $s, 1 \leq s \leq k$, we will mark vertices of $\lambda_{f}$ which are preimages of the point $c_{s}$ by the number $s$ (see Fig. 1). Define a star of $\lambda_{f}$ as a subset of edges of $\lambda_{f}$ consisting of edges adjacent to some non-marked vertex. If $U$ is a simply-connected domain such that $S \backslash\left\{c_{1}, c_{2}, \ldots, c_{k}\right\} \subset U$, then the set of stars of $\lambda_{P}$ may be naturally identified with the set of single-valued branches of $f^{-1}(z)$ in $U$ as follows: to the branch $f_{i}^{-1}(z), 1 \leq i \leq n$, corresponds the star $S_{i}$ such that $f_{i}^{-1}(z)$ maps bijectively the interior of $S$ to the interior of $S_{i}$.

Now in order to obtain a deformation of the integration path in (5) satisfying the requirements above we may just to deform the initial path inside of each disk 
$R \backslash \lambda_{f}$ avoiding poles of $f$ (see Fig. 2). If $\gamma$ is such a deformation and $c_{s, i}$ is a

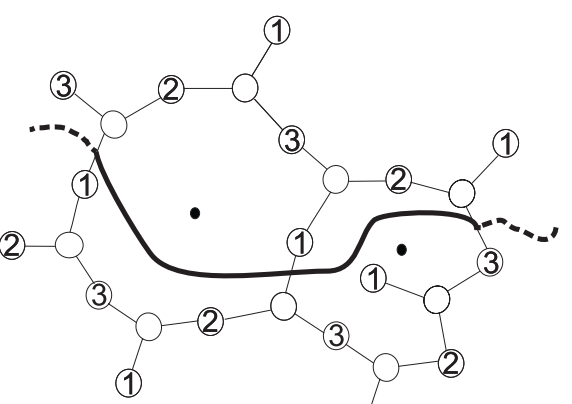

(1)

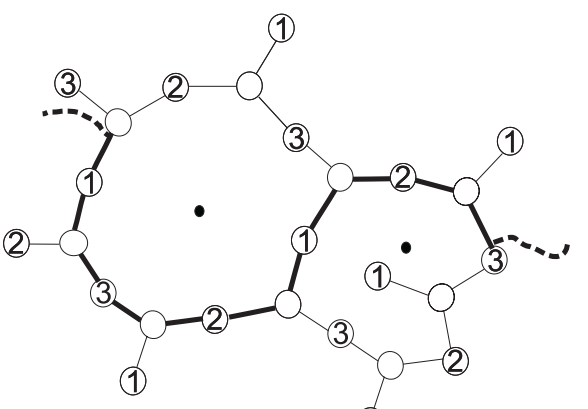

(1)

FiguRE 2

unique vertex of the star $S_{i}$ marked by the number $s$, then for $s, 1 \leq s \leq k$, we have:

$$
\varphi_{s}(z)=\sum_{i=1}^{n} n_{s i}\left(\frac{\omega}{d f}\right)\left(f_{i}^{-1}(z)\right),
$$

where $n_{s i}$ is a sum of "signed" appearances of the vertex $c_{s i}$ on the path $\gamma$. By definition, this means that an appearance is taken with the sign plus if the center of $S_{i}$ is followed by $c_{s i}$, and minus if $c_{s i}$ is followed by the center of $S_{i}$. For example, for the graph $\lambda_{f}$ shown on Fig. 1 and the path $\gamma \subset \lambda_{f}$ pictured by the fat line we have:

$$
\begin{gathered}
\varphi_{1}(z)=\left(\frac{\omega}{d f}\right)\left(f_{3}^{-1}(z)\right)-\left(\frac{\omega}{d f}\right)\left(f_{2}^{-1}(z)\right) \\
\varphi_{2}(z)=\left(\frac{\omega}{d f}\right)\left(f_{2}^{-1}(z)\right)-\left(\frac{\omega}{d f}\right)\left(f_{1}^{-1}(z)\right)+\left(\frac{\omega}{d f}\right)\left(f_{5}^{-1}(z)\right)-\left(\frac{\omega}{d f}\right)\left(f_{4}^{-1}(z)\right), \\
\varphi_{3}(z)=\left(\frac{\omega}{d f}\right)\left(f_{1}^{-1}(z)\right)-\left(\frac{\omega}{d f}\right)\left(f_{5}^{-1}(z)\right)+\left(\frac{\omega}{d f}\right)\left(f_{4}^{-1}(z)\right)-\left(\frac{\omega}{d f}\right)\left(f_{3}^{-1}(z)\right) .
\end{gathered}
$$

Clearly, since $\gamma$ is a closed loop, the above construction implies that $\sum_{j} n_{i j}=0$, so the cycles $\delta_{i}$ in $(13)$ are contained in $\widetilde{H}_{0}\left(f^{-1}(z), \mathbb{Z}\right)$ indeed. Furthermore, since $R \backslash \lambda_{f}$ is a disjoint union of disks each of which contains a single pole of $f$, the following statement holds.

Corollary 2.1. If the curve $\gamma$ is not homological to zero in $R$ with poles of $f$ removed, then the vanishing of moments (14) implies that there exists a non-zero cycle $\delta \in \widetilde{H}_{0}\left(f^{-1}(z), \mathbb{Z}\right)$ such that $\int_{\delta} \frac{\omega}{d f}=0$.

Observe that Theorem 2.1 and Corollary 2.1 remain true without the restriction that the set of poles of $\omega$ is contained in the set of poles of $f$ if to change the condition $J(t) \equiv 0$ to the condition that $J(t)$ is rational. Indeed, we always may find a polynomial $R$ such that the set of poles of the form $\widetilde{\omega}=R(f) \omega$ is contained in the set of poles of $f$. On the other hand, it follows from the definition of $J(t)$ as 
a generating function that the functions $J(\omega, f, \gamma, t)$ and $J(f \omega, f, \gamma, t)$ are related by the equality

$$
J(f \omega, f, \gamma, t)=J(\omega, f, \gamma, t) t-\int_{\gamma} \omega
$$

which implies inductively that the function $J(\omega, f, \gamma, t)$ is rational if and only if the function $J(\widetilde{\omega}, f, \gamma, t)$ does.

Further, observe that the above method may be applied also in the situation where the curve $\gamma$ is not closed and/or is not connected (see [15], Section 3 for the rational case which extends to the general case in the same way as above). Of coarse, if $\gamma$ is non-closed, then the condition $\sum_{j} n_{i j}=0$ for $\delta_{i}$ in (13) is not necessary true.

2.1. Solution of the moment problem in the case of generic position. Let $f$ be a meromorphic function on a compact Riemann surface $R, z_{0}$ be a fixed regular value of $f$, and $\Delta$ be the set of critical values of $f$. Recall that the monodromy group $G_{f}$ of the function $f$ is defined as the image of the homomorphism

$$
\pi_{1}\left(\mathbb{C} \backslash \Delta, z_{0}\right) \rightarrow \operatorname{Aut}\left(f^{-1}\left(z_{0}\right)\right),
$$

where $\operatorname{Aut}\left(f^{-1}\left(z_{0}\right)\right)$ is the full permutation group. Further, a meromorphic function $f: R \rightarrow \mathbb{C P}^{1}$ can be decomposed into a composition $f=p \circ q$ of holomorphic functions $q: R \rightarrow C$ and $p: C \rightarrow \mathbb{C P}^{1}$, where $C$ is another compact Riemann surface, if and only if the group $G_{f}$ has an imprimitivity system consisting of $d=\operatorname{deg} p$ blocks. Observe that if the set $\{1,2, \ldots, n\}$ is identified with the set $f^{-1}\left\{z_{0}\right\}$ then the set of blocks of the imprimitivity system corresponding to the decomposition $f=p \circ q$ has the form $\mathcal{B}_{i}=q^{-1}\left\{t_{i}\right\}, 1 \leq i \leq d_{1}$, where $\left\{t_{1}, t_{2}, \ldots, t_{d_{1}}\right\}=p^{-1}\left\{z_{0}\right\}$. Finally, notice that if $f=\widetilde{p} \circ \widetilde{q}$, where $\widetilde{q}: R \rightarrow \widetilde{C}, \widetilde{p}: \widetilde{C} \rightarrow \mathbb{C P}^{1}$, is an other decomposition of $f$ then the corresponding imprimitivity systems coincide if and only there exists an isomorphism $\mu: \widetilde{C} \rightarrow C$ such that

$$
p=\widetilde{p} \circ \mu^{-1}, \quad q=\mu \circ \widetilde{q} .
$$

In this case the decompositions $p \circ q$ and $\widetilde{p} \circ \widetilde{q}$ are called equivalent.

We say that meromorphic functions $f, g$ on a Riemann surface $R$ have a nontrivial common compositional right factor if there exists a Riemman surface $\widetilde{R}$, a holomorphic function $h: R \rightarrow \widetilde{R}$ of degree greater than one, and holomorphic functions $\widetilde{f}, \widetilde{g}: \widetilde{R} \rightarrow \mathbb{C P}^{1}$ such that $f=\widetilde{f} \circ h, g=\widetilde{g} \circ h$. The property of two functions $f, g$ to have a common compositional right factor may be expressed via the vanishing of some zero-dimensional Abelian integrals.

Proposition 2.1. Two meromorphic functions $f, g$ on a compact Riemann surface $R$ have a common compositional right factor if and only if there exists a cycle $\delta(z) \in \widetilde{H}_{0}\left(f^{-1}(z), \mathbb{Z}\right)$ of the form $f_{i}^{-1}(z)-f_{j}^{-1}(z)$ such that

$$
\int_{\delta(z)} g \equiv 0 .
$$

In particular, equality (17) holds for all $\delta(z) \in \widetilde{H}_{0}\left(f^{-1}(z), \mathbb{Z}\right)$ if and only if there exists a rational function $\widetilde{g}$ such that $g=\widetilde{g} \circ f$.

Proof. It is easy to see by the analytical continuation that, for a fixed index $i$, the indices $j$ satisfying the equality

$$
g\left(f_{i}^{-1}(z)\right)=g\left(f_{j}^{-1}(z)\right)
$$


form a block of an imprimitivity system $I$ with respect to the action of the monodromy group $G_{f}$ of $f$ on fibers of $f$. If (17) holds, then this block contains more than one element and therefore there exists $\widetilde{R}$ and a meromorphic function $h: R \rightarrow \widetilde{R}$ such that $f=\widetilde{f} \circ h$ and fibers of $h$ coincide with blocks of $I$. Furthermore, equalities (18) imply that the function $g$ is constant on fibers of $h$. Therefore, a function $\widetilde{g}=g \circ h^{-1}$ is well defined and satisfies the equality $g=\widetilde{g} \circ h$.

Notice that Proposition 2.1 permits to reduce the moment problem for a collection $f, \omega, \gamma, R$ to a similar problem for another collection $\widetilde{f}, \widetilde{\omega}, \widetilde{\gamma}, \widetilde{R}$ with $\operatorname{deg} \widetilde{f}<\operatorname{deg} f$ whenever the space generated by cycles (13), contains a simple cycle $f_{i}^{-1}(z)-f_{j}^{-1}(z)$, and in certain cases such a reduction may imply a complete solution of the initial problem see e.g. [10], [13], [15], [11], [3]. In the particular case $R=\mathbb{C P}^{1}$ the above Proposition is well known (see e.g. [10], Corollary 1 ) and follows easily from the Lüroth theorem. In particular, Proposition 2.1 permits to solve the moment problem for $f$ in a generic position.

Theorem 2.2. If the monodromy group $G_{f}$ of $f$ is the full symmetric group of $n=\operatorname{deg} f$ elements, then the vanishing of moments (14) implies that either $\gamma$ is homological to zero in $R$ with poles of $f$ removed, or there exists a rational function $Q$ such that $\omega=Q(f) d f$ and $f(\gamma)$ is homological to zero in $\mathbb{C P}^{1}$ with poles of $Q$ removed.

Proof. If $\gamma$ is not homological to zero in $R$ with poles of $f$ removed, then by Corollary 2.1 there exist integer numbers $\alpha_{1}, \alpha_{2}, \ldots, \alpha_{n}$ such that

$$
\sum_{i=1}^{n} \alpha_{i}\left(\frac{\omega}{d f}\right)\left(f_{i}^{-1}(z)\right)=0
$$

and $\sum_{i=1}^{n} \alpha_{i}=0$. The last equality implies that the numbers $\alpha_{1}, \alpha_{2}, \ldots, \alpha_{n}$ are not all equal between themselves. Let us assume that $\alpha_{1} \neq \alpha_{2}$.

Since $G_{f}$ is a full symmetric group it contains the transposition (12). Applying it to the equality (19) and subtracting we obtain the equality

$$
\left(\alpha_{1}-\alpha_{2}\right)\left(\left(\frac{\omega}{d f}\right)\left(f_{1}^{-1}(z)\right)-\left(\frac{\omega}{d f}\right)\left(f_{2}^{-1}(z)\right)\right)=0
$$

implying the equality

$$
\left(\frac{\omega}{d f}\right)\left(f_{1}^{-1}(z)\right)=\left(\frac{\omega}{d f}\right)\left(f_{2}^{-1}(z)\right) .
$$

Since the full symmetric group of $f$ is primitive, the function $f$ is indecomposable. Therefore, Proposition 2.1 applied to equality (20) implies that there exists a rational function $Q$ such that $\frac{\omega}{d f}=Q(f)$. Hence, moments (1) equal to the moments

$$
\int_{f(\gamma)} Q(z) z^{s} d z, \quad s \geq 0
$$

and the statement follows from the classical result of the complex analysis.

Notice that Theorem 2.2 also may be deduced from the characterization of doubly transitive groups via the structure of their irreducible subspaces over $\mathbb{C}$, see [10], [15] where this approach is used for rational $f$ and $g$. 


\section{VANishing of GenUs ZERO ABELIAN INTEGRAlS}

In this section we give necessary and sufficient conditions for an Abelian integral of dimension zero to vanish identically in the case where $R$ is the Riemann sphere and the functions $f$ and $g$ are polynomials. More precisely, we solve the following problem:

For a given polynomial $P$ of degree $n$ and a cycle $\delta(z) \in \widetilde{H}_{0}\left(P^{-1}(z), \mathbb{Z}\right)$ describe the polynomials $Q$ such that the associated Abelian integral

$$
I(z)=\int_{\delta(z)} Q=\sum_{i=1}^{n} m_{i} Q\left(P_{i}^{-1}(z)\right)
$$

vanishes identically.

In distinction with the previous section we will not assume that $\widetilde{H}_{0}\left(P^{-1}(z), \mathbb{Z}\right)$ is reduced. Thus $\delta(z)$ may be any expression of the form

$$
\delta(z)=v_{1} P_{1}^{-1}(z)+v_{2} P_{2}^{-1}(z)+\cdots+v_{n} P_{n}^{-1}(z)
$$

where $v_{i} \in \mathbb{Q}$. It is convenient to identify the cycle $\delta(z)$ with the vector

$$
\vec{\delta}=\left(v_{1}, v_{2}, \ldots, v_{n}\right)
$$

of $\mathbb{Q}^{n}$. Under such an identification the natural action of $G_{P}$ on $\widetilde{H}_{0}\left(P^{-1}(z), \mathbb{Z}\right)$ descends to an action of $G_{P}$ of $\mathbb{Q}^{n}$ defining a permutation representation of the group $G_{P}$

$$
\rho: G_{P} \rightarrow G L\left(\mathbb{Q}^{n}\right)
$$

The understanding of irreducible components of $\rho$ plays a crucial role in the solution of the problem above. Indeed, let $Z_{\delta}$ be the vector space consisting of polynomials $Q$ such that Abelian integral (21) vanishes identically, and $V_{\delta}$ be the minimal $\rho$-invariant vector subspace of $\mathbb{Q}^{n}$ containing the vector $\vec{\delta}$. Then it is easy to see by the analytical continuation that $\int_{\delta(z)} Q \equiv 0$ if and only if $\int_{\gamma(z)} Q \equiv 0$ for any $\gamma(z) \in \widetilde{H}_{0}\left(P^{-1}(z), \mathbb{Z}\right)$ such that $\vec{\gamma} \in V_{\delta}$. This implies that in order to describe $Z_{\delta}$ it is enough to solve the following three problems:

(1) First, describe all possible irreducible $G_{P}$-invariant subspaces of $\mathbb{Q}^{n}$.

(2) Second, for a given $\vec{\delta} \in \mathbb{Q}^{n}$ provide a method which allows to decompose the invariant space $V_{\delta}$ into a direct sum of irreducible $G_{P}$-invariant subspaces.

(3) Third, for a given irreducible $G_{P}$-invariant subspace $V$, describe the vector space $Z_{V}$ consisting of polynomials $Q$ such that $\int_{\delta(z)} Q \equiv 0$ for all $\delta \in V$.

The solution of these problems is implicitly contained in [14] as an ingredient of the proof of the so called polynomial moment problem. For the reader's convenience we present to the end of the section a self-contained proof, except of the classification irreducible $G_{P}$-invariant subspaces which is proved in [14] in a closed form.

3.1. Description of the irreducible $G_{P}$-invariant subspaces of $\mathbb{Q}^{n}$. Below we reproduce the description of $G_{P}$-invariant subspaces of $\mathbb{Q}^{n}$ obtained in [14]. More generally, we will describe $G$-invariant subspaces of $\mathbb{Q}^{n}$ for a permutation representation $G$ of an arbitrary permutation group $G \subset S_{n}$ containing a cycle of length $n$ (the monodromy group of a polynomial of degree $n$ always contains such a 
cycle which corresponds to a loop around infinity). Notice that if $P$ is decomposed into a composition of rational functions $P=A \circ B$, then in the corresponding equivalence class there exists a decomposition into a composition of polynomials and below we always will consider only such decompositions. In order to keep the correspondence between imprimitivity systems of $G_{P}$ and equivalence classes of decompositions of $P$ we need to modify the definition of equivalent decompositions correspondingly. Namely, we will call decompositions $P=A_{1} \circ W_{1}$ and $P=A_{2} \circ W_{2}$ equivalent if there exists a linear function $\nu$ such that $A_{2}=A_{1} \circ \nu, W_{2}=\nu^{-1} \circ W_{1}$. Abusing of notation, usually we will mean by a decomposition a corresponding equivalence class of decompositions.

Without loss of generality we may assume that a cycle of length $n$ contained in $G$ coincides with the cycle $(1, \ldots, n)$. Then it is easy to see that any imprimitivity system for $G$ coincides with the residue classes modulo $d$ for some $d \mid n$. In particular, this implies that any two decompositions $P=P_{1} \circ W_{1}$ and $P=P_{2} \circ W_{2}$ with $\operatorname{deg} P_{1}=\operatorname{deg} P_{2}$ are equivalent. For each $d \mid n$ we denote by $V_{d}$ the subspace of $\mathbb{Q}^{n}$ consisting of $d$-periodic vectors of the form

$$
\left(v_{1}, \ldots, v_{d}, v_{1}, \ldots, v_{d}, \ldots, v_{1}, \ldots, v_{d}\right) .
$$

It is easy to see that for given $d$ residue classes modulo $d$ form an imprimitivity system for $G$ if and only if the subspace $V_{d}$ is $G$-invariant.

Denote by $D(G)$ the set of all divisors of $n$ for which $V_{d}$ is $G$-invariant. In view of the above remarks $D(G)$ consists of numbers $d$ for which there exists a decomposition $P=A \circ W$ with $\operatorname{deg} A=d$. Notice that $D(G)$ is a lattice with respect to the operations $\wedge, \vee$, where $d \wedge \widetilde{d}:=\operatorname{gcd}(d, \widetilde{d})$ and $d \vee \widetilde{d}:=\operatorname{lcm}(d, \widetilde{d})$. Indeed, for an element $x \in X$ the intersection of two blocks containing $x$ and corresponding to $d, \widetilde{d} \in D(G)$ is a block which corresponds to $d \vee \widetilde{d}$. On the other hand, the intersection of two invariant subspaces $V_{d}, V_{\widetilde{d}}$ is an invariant subspace which is equal to $V_{d \wedge \widetilde{d}}$. We say that $d \in D(G)$ covers $\widetilde{d} \in D(G)$ if $\widetilde{d} \mid d, \widetilde{d}<d$, and there exists no $l \in D(G)$ such that $\widetilde{d}<l<d$ and $\widetilde{d}|l, l| d$.

Theorem 3.1. ([14]) Assume a permutation group $G \subseteq S_{n}$ contains the cycle $(12 \ldots n)$. Then each $G$-irreducible invariant subspace of $\mathbb{Q}^{n}$ has the form

$$
U_{d}:=V_{d} \cap\left(V_{d_{1}}^{\perp} \cap \ldots \cap V_{d_{\ell}}^{\perp}\right)
$$

where $d \in D(G)$ and $d_{1}, \ldots, d_{\ell}$ is a complete set of elements of $D(G)$ covered by $d$. The subspaces $U_{d}$ are mutually orthogonal and every $G$-invariant subspace of $\mathbb{Q}^{n}$ is a direct sum of some $U_{d}$ as above.

3.2. Decomposition of $V_{\delta}$ into a direct sum of irreducible subspaces. The vectors

$$
\vec{w}_{k}=\left(1, \varepsilon_{n}^{k}, \varepsilon_{n}^{2 k}, \ldots, \varepsilon_{n}^{(n-1) k}\right), \quad \varepsilon=e^{2 \pi i / n}, \quad 1 \leq k \leq n
$$

form an orthonormal basis of $\mathbb{C}^{n}$ with respect to the standard Hermitian scalar vector product in $\mathbb{C}^{n}$, and for any divisor $d$ of $n$ the vectors $\vec{w}_{k}$ for which $(n / d) \mid k$ form a basis of the complexification $V_{d}^{\mathbb{C}}$ of the subspace $V_{d}$. Furthermore, defining $\Psi_{d}, d \in D(G)$, as a subset of $\{1,2, \ldots, n\}$ consisting of numbers $r$ such that $n / d$ is a divisor of $r$ but for any element $\widetilde{d} \in D(G)$ covered by $d$ the number $n / \widetilde{d}$ is not a divisor of $r$, we see that the vectors $\vec{w}_{r}, r \in \Psi_{d}$, form a basis of $U_{d}^{\mathbb{C}}$. 
Theorem 3.2. The subspace $U_{d}, d \in D(G)$, is a component in the decomposition of the subspace $V_{\delta}$ into a sum of irreducible $G$-invariant subspaces of $\mathbb{Q}^{n}$, if and only if there exists a number $r \in \Psi_{d}$ such that $\left(\vec{\delta}, \vec{w}_{r}\right) \neq 0$.

Proof (cf. Proposition 4.1 in [14]). In view of Theorem 3.1, it is enough to show that $V_{\delta}$ is orthogonal to $U_{d}$ if and only if $\left(\vec{\delta}, \vec{w}_{r}\right)=0$ for any $r \in \Psi_{d}$. Let $V_{\delta}=\oplus U_{d_{j}}$ be the decomposition of $V_{\delta}$ into a sum of irreducible $G$-invariant subspaces. If $V_{\delta}$ is orthogonal to $U_{d}$, then $V_{\delta}$ should be orthogonal also to $U_{d}^{\mathbb{C}}$ implying that $\left(\vec{\delta}, \vec{w}_{r}\right)=0$ for all $r \in \Psi_{d}$. On the other hand, if $\left(\vec{\delta}, \vec{w}_{r}\right)=0$ for all $r \in \Psi_{d}$, then $\vec{\delta}$ is orthogonal to $U_{d}$. Therefore, $V_{\delta}$ is orthogonal to $U_{d}$ in view of the minimality of $V_{\delta}$.

3.3. Description of the spaces $Z_{U_{d}}$. The structure of $Z_{U_{d}}$ or, more generally, of any $Z_{\delta}$ is closely related to the compositional algebra of polynomials. For example, if $\delta(z) \in H_{0}\left(P^{-1}(z), \mathbb{Q}\right)$, then for any polynomial $Q$ of the form $Q=R \circ P$ we have

$$
\int_{\delta(z)} Q=R(z) \sum_{i=1}^{n} v_{i}
$$

implying that the corresponding integral vanishes whenever $\delta(z)$ is contained in the reduced homology group, or equivalently the vector $\vec{\delta}$ is contained in $V_{1}^{\perp}$.

Further, if $P=A \circ W, \operatorname{deg} A=d$, is a decomposition of $P$ corresponding to $d \in D\left(G_{P}\right)$, then for any branch $P_{i}^{-1}(z)$ of $P^{-1}(z)$ there exist a branch $W_{j}^{-1}(z)$ of $W^{-1}(z)$ and a branch $A_{k}^{-1}(z)$ of $A^{-1}(z)$ such that

$$
P_{i}^{-1}=W_{j}^{-1} \circ A_{k}^{-1} \text {. }
$$

Therefore, for any cycle $\delta(z) \in H_{0}\left(P^{-1}(z), \mathbb{Q}\right)$ and polynomial $Q$ we have

$$
\int_{\delta(z)} Q=\sum_{k=1}^{d}\left(\int_{\delta_{k, W}(z)} Q\right) \circ A_{k}^{-1},
$$

where $\delta_{k, W}(z) \in H_{0}\left(W^{-1}(z), \mathbb{Q}\right), d=\operatorname{deg} A$, and hence the integral $\int_{\delta(z)} Q$ vanishes identically whenever all the integrals $\int_{\delta_{k, W}(z)} Q, 1 \leq k \leq d$, do. In view of the above remark, this implies in particular that if $\delta(z) \in H_{0}\left(P^{-1}(z), \mathbb{Q}\right)$ is a cycle such that all cycles $\delta_{k, W}(z), 1 \leq k \leq d$, are in the reduced homology group $\widetilde{H}_{0}\left(W^{-1}(z), \mathbb{Q}\right)$, then $\int_{\delta(z)} Q$ vanishes identically for any polynomial $Q$ of the form $Q=B \circ W$.

In the following we always will assume that the numeration of roots $P_{i}^{-1}(z)$ of $P^{-1}(z)$ satisfies the requirement that the cycle in $G_{P}$ corresponding to a loop around infinity in $\mathbb{C}$ coincides with the cycle $(12 \ldots n)$. In particular, such a choice of the numeration yields that when $i$ in formula (26) runs a residues class modulo $d$, corresponding $j$ runs numbers from 1 to $n / d$ while $k$ remains fixed, implying that the cycles $\delta_{k, W}(z) \in H_{0}\left(W^{-1}(z), \mathbb{Q}\right)$ in $(27)$ are reduced if and only for and $k$, $1 \leq k \leq d$, the equality

$$
\left(\vec{\delta}, \vec{e}_{k, d}\right)=0
$$

holds, where $\vec{e}_{k, d}, 1 \leq k \leq d$, denotes a vector of $\mathbb{Q}^{n}$ with coordinates $v_{1}, v_{2}, \ldots, v_{n}$ such that $v_{i}=1$ if $i=k \bmod d$, and $v_{i}=0$ otherwise. Since vectors $\vec{e}_{j, d}, 1 \leq j \leq d$, obviously form a basis of $V_{d}$, we conclude that the cycles $\delta_{k, W}(z) \in H_{0}\left(W^{-1}(z), \mathbb{Q}\right)$ are reduced if and only if $\vec{\delta}$ is orthogonal to $V_{d}$.

Returning to the description of the space $Z_{U_{d}}$ observe that it always contains the space $Z_{V_{d}}$ in view of the inclusion $U_{d} \subset V_{d}$. Furthermore, if $\widetilde{d}$ if an element 
of $D\left(G_{P}\right)$ covered by $d$ and $P=\widetilde{A} \circ \widetilde{W}$ if a decomposition corresponding to $\widetilde{d}$, then, since $U_{d}$ is orthogonal to the subspace $V_{\widetilde{d}}$, the cycles $\delta_{k, \widetilde{W}}(z), 1 \leq k \leq \widetilde{d}$, are reduced. Therefore, for any such $\widetilde{d}$, the ring $\mathbb{C}[\widetilde{W}]$ of polynomials in $\widetilde{W}$ is contained in the space $Z_{U_{D}}$.

Theorem 3.3. Let $d$ be an element of $D\left(G_{P}\right)$. Furthermore, let $d_{1}, \ldots, d_{\ell}$ be a complete set of elements of $D(G)$ covered by $d$ and $P=A_{i} \circ W_{i}, 1 \leq i \leq \ell$, be the corresponding decompositions. Then

$$
Z_{U_{d}}=Z_{V_{d}}+\mathbb{C}\left[W_{1}\right]+\mathbb{C}\left[W_{2}\right]+\cdots+\mathbb{C}\left[W_{l}\right] .
$$

Proof (cf. Theorem 1.1 in [14]). In view of the above remarks, the right part of (29) is contained in $Z_{U_{d}}$. So, we only must establish the inverse inclusion.

First observe that the numeration of branches of $P^{-1}(z)$ implies that at points close enough to infinity the functions $Q\left(P_{i}^{-1}(z)\right), 1 \leq i \leq n$, may be represented by a converging Pusieux series

$$
Q\left(P_{i}^{-1}(z)\right)=\sum_{k=-q}^{\infty} s_{k} \varepsilon_{n}^{(i-1) k} z^{-\frac{k}{n}},
$$

where $q=\operatorname{deg} Q(z)$ and $\varepsilon_{n}=\exp (2 \pi i / n)$. Furthermore, substituting (30) to (21) we see that the integral $\int_{\delta(z)} Q$ vanishes identically if and only if for any $k \geq-q$ the equality

$$
\sum_{i=1}^{n} v_{i} s_{k} \varepsilon_{n}^{(i-1) k}=\left(\overline{\vec{\delta}}, \vec{w}_{k}\right) s_{k}=0
$$

holds. In particular, if $Q(z) \in Z_{U_{d}}, d \in D\left(G_{P}\right)$, then equalities

$$
\left(\overline{\vec{v}}, \vec{w}_{k}\right) s_{k}=0, \quad k \geq-q
$$

hold for any $\vec{v} \in U_{d}$. Moreover, they hold for any $\vec{v} \in U_{d}^{\mathbb{C}}$. Since $U_{d}^{\mathbb{C}}$ is generated by the set of vectors $\vec{w}_{r}, r \in \Psi_{d}$, and this set transforms to itself under the complex conjugation, this implies that if $Q(z) \in Z_{U_{d}}$, then for any $r \in \Psi_{d}$ the equality $s_{k}=0$ holds whenever $k \equiv r \bmod n$. Furthermore, clearly the inverse is also true. Similarly, $Q(z) \in Z_{V_{d}}$ if and only if $s_{k}=0$ for any $k$ such that $(n / d) \mid k$.

Assume now that $Q(z) \in Z_{U_{d}}$ and consider series (30). If $s_{k}=0$ for any $k$ such that $(n / d) \mid k$, then $Q(z) \in Z_{V_{d}}$. So, suppose that there exists $k$ such that $(n / d) \mid k$ but $s_{k} \neq 0$. Then $(n / \widetilde{d}) \mid k$ for some $\widetilde{d}$ covered by $d$ and we may assume that $\widetilde{d}=d_{1}$. Set

$$
\psi(z)=\sum_{\substack{k \geq-q \\ k \equiv 0 \bmod n / d_{1}}} s_{k} z^{-\frac{k}{n}},
$$

where $s_{k}, k \geq-q$, are coefficients of series (30). Clearly, we have:

$$
\left(\frac{n}{d_{1}}\right) \psi(z)=Q\left(P_{1}^{-1}(z)\right)+Q\left(P_{d_{1}+1}^{-1}(z)\right)+Q\left(P_{2 d_{1}+1}^{-1}(z)\right)+\ldots+Q\left(P_{n-d_{1}+1}^{-1}(z)\right) .
$$

Since the set of indices appearing in the right part of (32) is a block, the function $\psi(z)$ is invariant with respect to the subgroup of $G_{P}$ which stabilizes $P_{1}^{-1}(z)$. Therefore, by the main theorem of Galois theory, $\psi(z)$ is contained in the field $\mathbb{C}(z)\left(P_{1}^{-1}(z)\right)=\mathbb{C}\left(P_{1}^{-1}(z)\right)$ and hence $\psi(z)=R_{1}\left(P_{1}^{-1}(z)\right)$ for some rational function $R_{1}$. Moreover, $R_{1}$ is actually a polynomial since the right part of (32) may 
have a pole only at infinity. Further, since (32) implies that

$$
R_{1}\left(P_{1}^{-1}(z)\right)=R_{1}\left(P_{d_{1}+1}^{-1}(z)\right)=R_{1}\left(P_{2 d_{1}+1}^{-1}(z)\right)=\cdots=R\left(P_{n-d_{1}+1}^{-1}(z)\right),
$$

it follows from (26) that $R_{1}$ is constant on fibers of $W_{1}$. Reasoning now as in Proposition 2.1 we conclude that $R_{1}=S_{1} \circ W_{1}$ for some polynomial $S_{1}$ (cf. Lemma 4.3 in [14]).

Define a polynomial $T_{1}(z)$ by the equality

$$
T_{1}(z)=Q(z)-R_{1}(z) .
$$

Then by construction the Puiseux series of $T_{1}\left(P_{1}^{-1}(z)\right)$ contains no non-zero coefficients with indices which are multiple of $n / d_{1}$. If $T_{1}(z)$ is contained in $Z_{W}$, then

$$
Q(z)=T_{1}(z)+S_{1}\left(W_{1}(z)\right)
$$

and we are done. Otherwise arguing as above we may find polynomials $R_{2}, S_{2}$ such that $R_{2}=S_{2} \circ W_{2}$ and the Puiseux expansion of $T_{2}\left(P_{1}^{-1}(z)\right)$, where

$$
T_{2}(z)=T_{1}(z)-S_{2}(z),
$$

contains no non-zero coefficients whose indices are multiple of $n / d_{1}$ or $n / d_{2}$. It is clear that continuing this process we eventually will arrive to some $T_{s}(z)$ which is contained in $Z_{W}$ and therefore to a representation

$$
Q(z)=T_{s}(z)+S_{1}\left(W_{1}(z)\right)+S_{2}\left(W_{2}(z)\right)+\cdots+S_{l}\left(W_{l}(z)\right) .
$$

In view of Theorem 3.3 in order to complete the description of the space $Z_{U_{d}}$ we only must describe the space $Z_{V_{d}}$. Observe that the vectors $\vec{e}_{j, d}, 1 \leq j \leq d$, defined above satisfies the equality

$$
\left(\vec{e}_{j, d}, \vec{w}_{k}\right)=\varepsilon_{n}^{k(j-1)}\left(\vec{e}_{1, d}, \vec{w}_{k}\right) .
$$

Therefore, in order to check that (31) holds for any $\vec{v} \in Z_{V_{d}}$ it is enough to check that it holds for one single vector $\vec{e}_{1, d}$. In other words, the space $Z_{V_{d}}$ consists of polynomials $Q(z)$ satisfying the equality

$$
Q\left(P_{1}^{-1}(z)\right)+Q\left(P_{d+1}^{-1}(z)\right)+Q\left(P_{2 d+1}^{-1}(z)\right)+\ldots+Q\left(P_{n-d+1}^{-1}(z)\right) \equiv 0 .
$$

Furthermore, if $P=A \circ W$ is a decomposition corresponding to $d \in D\left(G_{P}\right)$, then in view of (26) equality (33) reduces to the equality

$$
Q\left(W_{1}^{-1}(z)\right)+Q\left(W_{2}^{-1}(z)\right)+Q\left(W_{3}^{-1}(z)\right)+\ldots+Q\left(W_{n / d}^{-1}(z)\right) \equiv 0 .
$$

The Newton formulae imply that whenever $\operatorname{deg} Q<\operatorname{deg} W$ the sum in the left hand side of (34) is a constant. Therefore, if $\mu_{i}=W_{i}^{-1}(c), i=1,2, \ldots, n / d$, for some generic $c$, then the relation

$$
Q\left(\mu_{1}\right)+Q\left(\mu_{2}\right)+\cdots+Q\left(\mu_{n / d}\right)=0
$$

describes the vector space of polynomials satisfying (34). Furthermore, for $Q(z)$ of arbitrary degree it is easy to see, using $W$-adic decomposition, that the sum in (34) is a polynomial, and that $Q(z)$ satisfies (34) if and only if all coefficients in its $W$-adic decomposition satisfy it. 
3.4. Corollaries. In this subsection we discuss some particular cases of the above results which may be useful for applications.

Proposition 3.1. Let $P$ be an indecomposable polynomial. If an Abelian integral $\int_{\delta(z)} Q$, where $Q$ is a non-zero polynomial and $\delta(z) \in H_{0}\left(P^{-1}(z), \mathbb{Q}\right)$, vanishes identically, then either $Q$ is a polynomial in $P$ and the cycle $\delta(z)$ is reduced, or there exists a rational number a such that

$$
\delta(z)=a\left(P_{1}^{-1}(z)+P_{2}^{-1}(z)+\cdots+P_{n}^{-1}(z)\right) .
$$

Proof. It follows from the assumption $Q(z) \not \equiv 0$ that the subspace $V_{\delta}$ does not coincide with whole $\mathbb{Q}^{n}$. Therefore, by Theorem 3.1 either $V_{\delta}=U_{1}$ or $V_{\delta}=U_{n}$. Obviously in the first case (35) holds while in the second $\delta(z)$ is reduced. Furthermore, by Theorem 3.3 in the second case $Q$ is contained in $Z_{U_{n}}=Z_{V_{n}}+\mathbb{C}[P]$. However, since $V_{n}=\mathbb{Q}^{n}$, the space $Z_{V_{n}}$ is trivial and therefore $Q$ is a polynomial in $P$.

Notice that the conclusion of Proposition 3.1 holds for any polynomial $P(z)$ in generic position since decomposable polynomials obviously form a proper algebraic subset in the set of all polynomials of degree $n$. Notice also that in order to prove Proposition 3.1 one can use instead of Theorem 3.1 a classical result, relating doubly transitivity of a group with the structure of its permutation representation over $\mathbb{C}$, and Schur theorem, relating doubly transitivity and primitivity for group containing a transitive cyclic subgroup (see [10] for such an approach).

The conclusion similar to the one in Proposition 3.1 is true for arbitrary $P$ if to impose some limitations on $\delta(z)$.

Proposition 3.2. If an Abelian integral $\int_{\delta(z)} Q$, where $Q$ is a non-zero polynomial and $\delta(z) \in H_{0}\left(P^{-1}(z), \mathbb{Q}\right)$, vanishes identically, and for any $d \in D\left(G_{P}\right), d \neq 1$, there exists $r \in \Psi_{d}$ such that $\left(\vec{\delta}, \vec{w}_{r}\right) \neq 0$, then $Q$ is a polynomial in $P$ and the cycle $\delta(z)$ is reduced.

Proof. It follows from Theorem 3.1 and Theorem 3.2 that $V_{\delta}=U_{1}^{\perp}$. In particular, the cycle $\delta(z)$ is reduced. Furthermore, $V_{\delta}$ contains vectors $e_{i}-e_{j}, 1 \leq i, j \leq n$, implying that

$$
Q\left(P_{1}^{-1}(z)\right)=Q\left(P_{2}^{-1}(z)\right)=\cdots=Q\left(P_{n}^{-1}(z)\right) .
$$

Now the statement follows from Proposition 2.1.

A weaker version of Proposition 3.2 can be formulated as follows

Proposition 3.3. If an Abelian integral $\int_{\delta(z)} Q$, where $Q$ is a non-zero polynomial and $\delta(z) \in H_{0}\left(P^{-1}(z), \mathbb{Q}\right)$, vanishes identically, and there exists $r \in \Psi_{n}$ such that $\left(\vec{\delta}, w_{r}\right) \neq 0$, then $Q(z)$ may be represented in the form

$$
Q(z)=S_{1}\left(W_{1}(z)\right)+S_{2}\left(W_{2}(z)\right)+\cdots+S_{l}\left(W_{l}(z)\right)
$$

where $S_{1}, S_{2}, \ldots, S_{l}$ are polynomials and $W_{1}, W_{2}, \ldots W_{l}$ are polynomial compositional right factors of $P(z)$.

Proof. It follows from Theorem 3.2 that $V_{\delta}$ contains $U_{n}$. Therefore, $Z_{\delta} \subseteq Z_{U_{n}}$ and the statement follows from Theorem 3.3. 
Notice that in general it is not true that for any polynomial $W_{i}$ appearing in representation (37) the corresponding cycles

$$
\delta_{k, W_{i}}(z) \in \widetilde{H}_{0}\left(W_{i}^{-1}(z), \mathbb{Q}\right)
$$

are reduced. On the other hand, if it is true, then the theorem is equivalent to the statement that the integral $\int_{\delta(z)} Q$ vanishes if and only if it is a sum of pull-backs of integrals along cycles homological to zero. Therefore, it is of interest to give conditions providing that there exists a representation (37) such that all cycles (38) are reduced.

Proposition 3.4. Assume that an Abelian integral $\int_{\delta(z)} Q$, where $Q$ is a non-zero polynomial and $\delta(z) \in H_{0}\left(P^{-1}(z), \mathbb{Q}\right)$ vanishes identically. Furthermore, assume that there exist $d_{1}, d_{2}, \ldots d_{l} \in D\left(G_{P}\right)$ such that $\left(\vec{\delta}, w_{r}\right)=0$ if and only if $r$ is a divisor of one of $d_{1}, d_{2}, \ldots d_{l}$. Then $Q(z)$ may be represented in the form (37) where all cycles (38) are reduced.

Proof. Since, the condition of the theorem implies that $V_{\delta}$ coincides with the orthogonal complement to the sum of $V_{n / d_{1}}, V_{n / d_{2}}, \ldots, V_{n / d_{l}}$ in $\mathbb{Q}^{n}$, the proof is obtained by an obvious modification of the proof of Theorem 3.3.

Remark. The results similar to Propositions 3.3, 3.4 (without the solution of the general problem) were obtained in the recent preprint [1] (Thereom 2.2) where they also were deduced from Theorem 3.1 by the method of [14]. However, in [1] they seem to be formulated not in their full generality. For example, the second part of Theorem 2.2 which is an analog of our Proposition 3.3 is formulated under an unnecessary condition that $r \in \Psi_{n}$ for which $\left(\vec{w}, \vec{w}_{r}\right) \neq 0$ is coprime with $n$.

3.5. Polynomial moment problem on a system of intervals with weights. Recall that the polynomial moment problem, recently solved in [14], asks about conditions implying that a polynomial $Q$ satisfies the system of equations

$$
\int_{a}^{b} P^{i} d Q=0, \quad i \geq 0
$$

where $P$ is a given polynomial and $a, b$ are fixed complex numbers. It was shown in [14] that any solution $Q$ of this problem has the form (37) where all $W_{i}$ satisfy the condition $W_{i}(a)=W_{i}(b)$. In the above notation the approach of [14] may be summarized as follows. First, to construct, starting from the collection $P, a, b$, a collection of cycles $\delta_{j}(z), 1 \leq j \leq s$, in $\widetilde{H}_{0}\left(P_{i}^{-1}(z), \mathbb{Q}\right)$ such that $(39)$ holds if and only if

$$
\int_{\delta_{i}(z)} Q=0, \quad 1 \leq j \leq s .
$$

Then to show that the corresponding invariant subspace generated by the cycles $\delta_{j}(z), 1 \leq j \leq s$, always contains a vector $\vec{v}$ such that $\left(\vec{v}, w_{r}\right) \neq 0$ for some $r$ coprime with $n$ (this was done in [13] by means the so-called "monodromy lemma") and, finally, to use Proposition 3.3 repeatedly.

It is not hard to see that the results of the current section may be interpreted as a solution of the polynomial moment problem "on a system of intervals with weights". More precisely, for any collection consisting of a polynomial $P$, complex numbers $a_{i}, b_{i}, 1 \leq i \leq l$, and rational numbers $c_{i}, 1 \leq i \leq l$, using approach of Section 1 (see also [14], [15] where more attention to non-closed curves is given) 
one can construct a finite collection of cycles $\delta_{j}(z), 1 \leq j \leq s$, in $\widetilde{H}_{0}\left(P_{i}^{-1}(z), \mathbb{Q}\right)$ such that the equalities

$$
c_{1} \int_{a_{1}}^{b_{1}} P^{i} d Q+c_{2} \int_{a_{2}}^{b_{2}} P^{i} d Q+\cdots+c_{l} \int_{a_{l}}^{b_{l}} P^{i} d Q=0, \quad i \geq 0,
$$

hold if and only if equalities (40) hold. Since the results of this section provide a description of $Q$ satisfying (40), we obtain therefore a description of solutions of (41). Notice however that since $G_{P}$-invariant subspace $V$ of $\mathbb{Q}^{n}$ generated by the vectors $\vec{\delta}_{j}, 1 \leq j \leq s$, obtained from (41) may be arbitrary we can not expect to have such a good description of solutions as for problem (39).

As a simple illustration, let us take $P$ equal to $T_{6}$, where $T_{n}$ denotes the $n$th Chebyshev polynomial, $T_{n}(\cos \varphi)=\cos (n \varphi)$. It is easily seen that $T_{n}$ has only two finite critical values and that the corresponding constellation has the form shown on the Fig. 3,

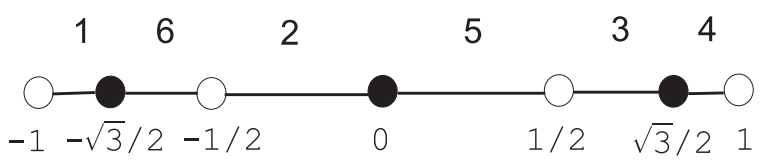

FigURE 3

where the "middle" vertices of stars are omitted and the numeration of stars is chosen in such a way that a permutation at infinity coincides with the cycle (123456) (see e.g. [12]).

If we are searching for solutions of the moment problem

$$
\int_{-\sqrt{3} / 2}^{\sqrt{3} / 2} T_{6}^{i} d Q=0, \quad i \geq 0,
$$

on a single segment $[-\sqrt{3} / 2, \sqrt{3} / 2]$, we arrive to the vanishing problem for the Abelian integral

$$
\delta(z)=Q\left(T_{6,6}^{-1}(z)\right)-Q\left(T_{6,2}^{-1}(z)\right)+Q\left(T_{6,5}^{-1}(z)\right)-Q\left(T_{6,3}^{-1}(z)\right)
$$

where by $T_{6, i}^{-1}(z), 1 \leq i \leq 6$, are denoted the branches of $T_{6}^{-1}$ (although we should obtain a cycle for each critical value, it is easy to see that, since we have only two critical values, the corresponding cycles are proportional). Clearly, the corresponding vector $(0,-1,-1,0,1,1) \in \mathbb{Q}^{6}$ is orthogonal to both $V_{2}$ and $V_{3}$ implying that $V_{\delta}=U_{6}$. Therefore, by Theorem 3.3 any solution of (42) has the form

$$
Q(z)=A\left(T_{3}(z)\right)+B\left(T_{2}(z)\right),
$$

where $A, B \in \mathbb{C}[z]$.

On the other hand, the "generalized" moment problem

$$
\int_{-1}^{-1 / 2} T_{6}^{i} d Q-\int_{-1 / 2}^{1 / 2} T_{6}^{i} d Q+\int_{1 / 2}^{1} T_{6}^{i} d Q=0, \quad i \geq 0,
$$

leads to the vanishing problem for the Abelian integral

$\delta(z)=Q\left(T_{6,1}^{-1}(z)\right)-Q\left(T_{6,2}^{-1}(z)\right)+Q\left(T_{6,3}^{-1}(z)\right)-Q\left(T_{6,4}^{-1}(z)\right)+Q\left(T_{6,5}^{-1}(z)\right)-Q\left(T_{6,6}^{-1}(z)\right)$. 
One can check that in this case the corresponding invariant subspace $V$ of $\mathbb{Q}^{6}$ coincides with $U_{3}$ and Theorem 3.3 implies that any solution of (43) has the form

$$
Q(z)=A\left(T_{6}(z)\right)+B(z),
$$

where $A$ is an arbitrary polynomial and $B$ is a polynomial such that

$$
B\left(T_{3,1}^{-1}(z)\right)+B\left(T_{3,2}^{-1}(z)\right)+B\left(T_{3,3}^{-1}(z)\right) \equiv 0 .
$$

\section{VANishing OF hyPERELLIPTIC ABelian integrals}

Let $f \in \mathbb{C}[x]$ be a polynomial and $\Gamma_{t}=\left\{(x, y) \in \mathbb{C}^{2}: y^{2}-f(x)=t\right\}$ a family of hyperelliptic curves. Consider the Abelian integral

$$
I(t)=\int_{\gamma(t)} \omega
$$

where $\omega=P(x, y) d x+Q(x, y) d y$ is a polynomial one form, and $\gamma(t) \in H_{1}\left(\Gamma_{t}, \mathbb{Z}\right)$ is a continuous family of 1-cycles.

The purpose of this section is to determine necessary and sufficient conditions for the Abelian integral $I$ to be single valued, polynomial, or rational function. These three conditions are in fact equivalent. Indeed, $I(t)$ is a function of moderate growth, with a bounded modulus in any sector, centered at a singularity. Thus $I(t)$ is single-valued if and only if it is a rational, in fact polynomial function.

4.1. Vanishing and the moment problem. The derivatives of $I$ can be seen as moments on a Riemann surface and this permits to apply the results of the preceding section. Indeed, every polynomial one-form $\omega$ can be written as

$$
\omega=k(x) y d x+d A+B d\left(y^{2}-f(x)\right), \quad A, B \in \mathbb{C}[x, y], k \in \mathbb{C}[x] .
$$

Therefore,

$$
I(t)=\int_{\gamma(t)} k(x) y d x, \quad I^{\prime}(t)=\frac{1}{2} \int_{\gamma(t)} \frac{k(x) d x}{y}
$$

and more generally

$$
I^{(k+1)}(t)=(1 / 2)(-1 / 2)(-3 / 2) \ldots(-k+1 / 2) \int_{\gamma(t)} \frac{k(x)}{y^{2 k+1}} d x, k \geq 0 .
$$

Thus,

$$
I^{(k+1)}(0)=m_{k}=(1 / 2)(-1 / 2)(-3 / 2) \ldots(-k+1 / 2) \int_{\gamma(0)} g^{k+1} \omega
$$

where

$$
\omega=k(x) y d x, \quad g=\frac{1}{f},
$$

implying that the Abelian integral $I(t)$ vanishes identically if and only if the moments $\int_{\gamma(0)} g^{k} \omega, k \geq 0$, vanish. Furthermore, if to replace $\omega$ by $g^{k} \omega$, then, for $k$ sufficiently big, the set of poles of $g^{k} \omega$ will be a subset of the set of poles of $g$ and the results of Section 1 apply.

The zero-dimensional integrals described in Theorem 2.1 take the form

$$
\varphi_{i}(z)=\int_{\delta_{i}} \frac{\omega}{f^{k} d f}=\int_{\delta_{i}(z)} \frac{k(x)}{f^{(2 k-1) / 2} f^{\prime}(x)}=z^{-(2 k-1) / 2} \frac{d}{d z} \int_{\delta_{i}(z)} K(x)
$$


where $K(x)=\int k(x) d x$ is a primitive of $k$, and the zero-cycles $\delta_{i}$ are constructed from the constellation $\lambda_{f}=f^{-1}(S)$ as explained in section 2. The above gives necessary and sufficient conditions for the moments $m_{i}, i \geq k$ to vanish or, equivalently, for $I(t)$ to be a polynomial. Thus, we have proved

Theorem 4.1. The Abelian integral (44) is a rational function if and only if the o-dimensional integrals

$$
\int_{\delta_{i}(z)} K(x), i=1,2, \ldots, k
$$

are identically constant.

Remark. Consider the polynomial $f(x)=\left(x^{2} / 2-1\right)^{2}$ as on fig. 8 bellow and the family of 1-cycles $\gamma(t)$, represented on the $x$-plane by a big loop surrounding the four roots of $f(x)+t$, on the family of elliptic curves $\Gamma_{t}$. Further, consider the complete elliptic integral $I(t)=\int_{\gamma(t)} x y d x$. A simple computation shows that the associated 0 -dimensional Abelian integral is $\int_{\delta(z)} x^{2}$, where $\delta(z)=x_{1}(z)+x_{2}(z)+x_{3}(z)+x_{4}(z)$, $f\left(x_{i}(z)\right) \equiv z$. On the other hand

$$
I^{\prime}(t)=\int_{\gamma(t)} \frac{x d x}{2 y}
$$

is a complete elliptic integral of third kind, and $\gamma(t)$ is homologous to a small loop around one of the two "infinite" point of the affine curve $\Gamma_{t}$. The conclusion is that $I^{\prime}(t)$ is a residue, in fact a non zero constant. The Abelian integral $I(t)$ is therefore linear in $t$. This example shows that the claim of Theorem 4.1 can not be improved.

Note that the zero-cycles $\delta_{i}(z)$ are by no means unique, they depend on the mutual position of the segments $\left[c_{0}, c_{i}\right]$. If all the zero-cycles $\delta_{i}(z)$ are in the orbit of a given cycle $\delta_{i_{0}}$, obtained after a continuation with respect to $z$, then the vanishing of $\varphi_{i_{0}}$ implies the vanishing of all the $\varphi_{i}$, and hence of all the moments. Finally, the orbit of a given $\delta_{i_{0}}$ may contain other cycles, more suitable for our purposes. In the next subsection we propose an alternative construction of such a cycle, by using a residue calculus. As we shall see, this will be more natural for the applications.

4.2. The Cauchy integral related to $I$. In this section we give an alternative computation of a convenient necessary condition for the identical vanishing of the Abelian integral $I(t)$, defined in (44), (45). Our result will hold under the additional assumption that there is a path along which the cycle $\gamma(t)$ vanishes. More precisely, let $\gamma(t) \subset \Gamma_{t}$ be a continuous family of closed continuous curves defined in a neighborhood of some regular value $t_{0}$ of $f$. Consider a path

$$
[0,1] \rightarrow \mathbb{C}: s \mapsto t(s)
$$

such that $s(0)=t_{0}, s(1)=t_{1}, t(s)$ is a regular value of $f$ for $0 \leq s<1$, and $t_{1}$ is a singular value of $f$. We shall say that the continuous family of closed loops $\gamma(t)$ vanishes along the path (47) if it can be extended to a continuous family of loops along this path such that $\gamma\left(t_{1}\right)$ is homologous to zero on the singular affine curve $\Gamma_{t_{1}}$. This implies in particular that $I\left(t_{1}\right)=0$ as well as that the corresponding zero-dimensional Abelian integral vanishes at $t_{1}$.

Without loss of generality we suppose that $y$ restricted to $\gamma\left(t_{0}\right)$ does not vanish. Then, for all $(t, z)$ such that $|z|$ and $\left|t-t_{0}\right|$ are sufficiently small, the Cauchy type 
integral

$$
J_{t}(z)=\int_{\gamma(t)} \frac{k(x) y}{y^{2}-z} d x, \quad z \sim 0
$$

is well defined and analytic in $t, z$. The definition of $J_{t}(z)$ is illustrated on fig.4,

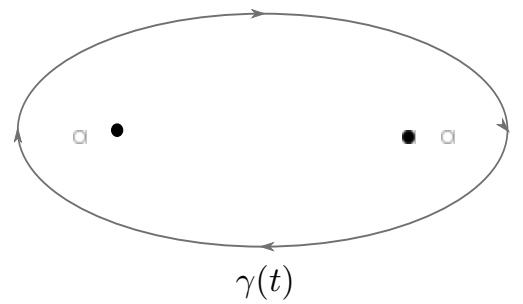

Figure 4 . The definition of the Cauchy type integral $J_{t}(z)$.

where a closed loop $\gamma(t)$ projected on the $x$-plane is shown, which makes one turn around two roots of the polynomial $f(x)+t$. The roots of $f(x)+t$ are represented by small black bullit circles, while the roots of $f(x)+t-z$ by small empty circles. Note that in section 2 we supposed that $z \sim \infty$, while in this section that $z \sim 0$, and this is essential for what follows.

Since $I^{\prime}(t)=\frac{1}{2} J_{t}(0), J_{t}(z)$ is a deformation of the Abelian integral $I^{\prime}(t)$. At the same time, for a fixed $t, J_{t}(z)$ is a generating function of the moments $I^{k}(t), k \geq 1$, in the sense of section 2 and

$$
J_{t}(z)=J(z)
$$

where the Riemann surface $R=\Gamma_{t}$ depends on the parameter $t$. For $(x, y) \in \gamma(t)$ and $|z|$ sufficiently small the series

$$
\sum_{k=0}^{\infty}\left(\frac{z}{y^{2}}\right)^{k}
$$

converges uniformly and hence

$$
\begin{aligned}
J_{t}(z) & =\int_{\gamma(t)} \frac{k(x) y}{y^{2}\left(1-\frac{z}{y^{2}}\right)} d x \\
& =\int_{\gamma(t)} \frac{k(x)}{y} d x+z \int_{\gamma_{0}} \frac{k(x)}{y^{3}} d x+z^{2} \int_{\gamma_{0}} \frac{k(x)}{y^{5}} d x+\ldots
\end{aligned}
$$

Taking into consideration (46) we conclude

Proposition 4.1. For every regular value $t$ of $f$ the equalities

$$
\left(\begin{array}{c}
-1 / 2 \\
k
\end{array}\right) \frac{d^{k}}{d z^{k}} J_{t}(0)=2 I^{(k+1)}(t), k=0,1,2 \ldots
$$

hold.

The above Proposition implies the following corollary.

Corollary 4.1. The Abelian integral $I^{\prime}(t)$ vanishes identically, if and only if the Cauchy type integral $J_{t}(z)$ vanishes identically.

The main advantage of using $J_{t}(z)$ instead of $I^{\prime}(t)$ is the possibility to extend it analytically with respect to $z$. The result is a function algebraic in $z$. 
Proposition 4.2 ([11]). For every fixed regular value $t$ the Cauchy type integral $J_{t}(z)$ extends to an algebraic function in $z$ with singularities at $z=0$ and at the critical values of $f$.

Indeed, for a fixed regular $t, J_{t}(z)$ allows for an analytic continuation along any path which does not contain critical values of $f-t$ or the value $z=0$. In a neighborhood of a critical value of $f-t$ or at $z=0$, the Cauchy theorem implies that, up to an addition of a holomorphic function, $J_{t}(z)$ is a linear combination of residues of $\frac{k(x) y}{y^{2}-z} d x$ at the roots $f_{i}^{-1}(z-t)$ of $f(x)+t-z$. Thus, $J_{t}(z)$ is a function of moderate growth in $z$ with a finite number of branches, and hence is algebraic in $z$.

Our next goal is to extend analytically $J_{t}(z)$ in a neighborhood of $\left(t_{1}, 0\right)$ under the condition that $t_{1}$ is a critical value of $f$. To simplify the notation put $t_{1}=0$, $f(0)=0$. Consider the domain

$$
D_{\delta}=\{(t, z):|t|<\delta,|z|<\delta, t \neq z, t \neq 0, z \neq 0\}
$$

and assume that $\delta>0$ is so small that $t=0$ is the only critical value of $f$ in the disc $\{t:|t|<2 \delta\}$. Take some $(t, 0) \in D_{\delta}$ and consider the germ of the analytic function $J=J_{t}(z)$ in a neighborhood of this point.

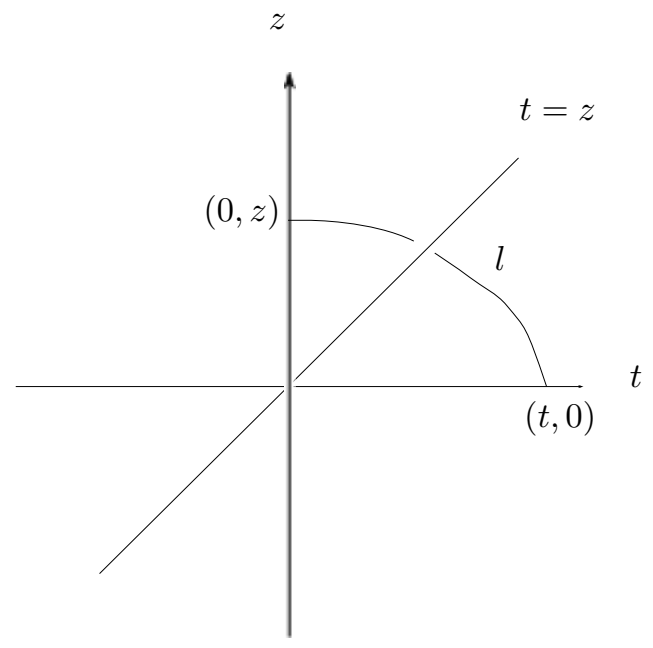

Figure 5. The domain $D_{\delta}$.

Proposition 4.3. The germ of $J=J_{t}(z)$ at $(t, 0) \in D_{\delta}$ allows for an analytic continuation along any path starting at $(t, 0)$ and contained in $D_{\delta}$.

Indeed, the affine curve $\Gamma_{t}$ is regular, provided that $t \neq 0$, and the differential $\frac{k(x) y}{y^{2}-z} d x$ has simple poles if and only if $z \neq t, z \neq 0$. Therefore the closed curve $\gamma(t)$ can be deformed in a way to avoid these simple poles.

Although the function $J_{t}(z)$ might be not analytic in $t$ near the line $t=0$, it has a finite limit there which we compute next. For this purpose, let $l$ be a closed smooth path connecting the point $(t, 0)$ to $(0, z), t, z \neq 0$, and contained in $D_{\delta}$ 
(except the ends), see fig. 5. Suppose that the homology class of the limiting loop $\gamma(0) \subset \Gamma_{0}$ is zero and hence is a linear combination of vanishing cycles.

Theorem 4.2. If $\gamma(0) \subset \Gamma_{0}$ is homologous to zero, then the limiting value of $J_{t}(z)$ at $(0, z)$ along $l$ is a zero-dimensional Abelian integral

$$
J_{0}(z)=2 \pi \sqrt{-z} \frac{d}{d z} \int_{\delta(z)} K(x)
$$

where $K(x)$ is a primitive of $k(x), \delta(z)=\sum_{i} n_{i} f_{i}^{-1}(z), f_{i}^{-1}(z)$ are the roots of the polynomial $f(x)-z$, and the numbers $n_{i}$ depend only on the homology class represented by the loop $\gamma(0)$ in $H_{1}\left(\check{\Gamma}_{0}, \mathbb{Z}\right), \check{\Gamma}_{0}=\left\{(x, y): y^{2}=f(x), f(x) \neq z\right\}$.

Corollary 4.2. If $I(t)=\int_{\gamma(t)} \omega \equiv 0$ then $\int_{\delta(z)} K \equiv 0$

Corollary 4.3. According to Proposition 4.1, if $I^{\prime}(t)=0$ for some regular $t$, then the multiplicity of this zero is the same as the multiplicity of $J_{t}(z)$ with respect to $z$ at $z=0$. In the particular case where $t=0$ is a Morse critical point, the Abelian integral $I^{\prime}$ is analytic at $t=0$, and the multiplicity of the zero of $I^{\prime}$ at $t=0$ is just the multiplicity of the zero of the analytic function $J_{0}(z)$ at $z=0$. Thus, the multiplicity of the one-dimensional Abelian integral at a Morse critical point equals essentially the corresponding multiplicity of the 0-dimensional Abelian integral.

Proof of Theorem 4.2. We can deform the loop $\gamma(t)$ along the interior of the path $l$ in a way to avoid the poles of $\frac{k(x) y}{y^{2}-z} d x$. Taking the limit $t \rightarrow 0$ along $l$ we obtain that $\gamma(0)$ is homologous to a sum of closed loops around the poles of $\frac{k(x) y}{y^{2}-z} d x$, as it is shown on fig. 6. Therefore,

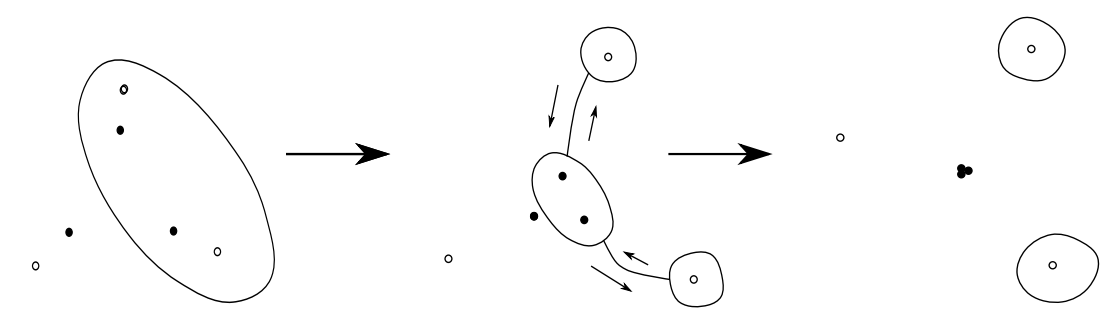

FIgURE 6. Computing the limit of $\gamma(t)$ at a singular value.

$$
\begin{aligned}
J_{0}(z) & =2 \pi \sqrt{-1} \sum_{i} n_{i} \operatorname{Res}_{f_{i}^{-1}(z)} \frac{k(x) y}{f(x)-z} d x \\
& =2 \pi \sqrt{-z} \sum_{i} \pm n_{i} \frac{k\left(f_{i}^{-1}(z)\right)}{f^{\prime}\left(f_{i}^{-1}(z)\right)} \\
& =2 \pi \sqrt{-z} \int_{\delta(z)} \frac{k(x)}{f^{\prime}(x)} \\
& =2 \pi \sqrt{-z} \frac{d}{d z} \int_{\delta(z)} K(x) .
\end{aligned}
$$

Computation of the reduced $\mathbf{0}$-cycle $\delta(z)$. For simplicity, suppose that $\gamma(t)$ vanishes as $t$ tends to 0 at the origin $(0,0)$. Thus $\gamma(t)$ is a linear combination of 
cycles vanishing at $(0,0)$. The standard basis of such cycles can be described as follows. Let $f_{i}^{-1}(t), i=1,2, \ldots, n$ be the roots of the polynomial $f(x)+t$ which tend to 0 as $t$ tends to 0 , ordered cyclically with respect to the monodromy action. We denote by $\gamma_{i j}(t) \subset \Gamma_{t}$ a simple closed loop which is projected to the segment $\left[f_{i}^{-1}(t), f_{j}^{-1}(t)\right]$. The loops $\gamma_{i, i+1}(t), i=1,2, \ldots, n-1$ form a basis of the local homology group of the Milnor fiber of $y^{2}-f(x)$. We fix the orientations of these cycles by the convention

$$
\gamma_{i, i+1} \cdot \gamma_{i+1, i+2}(t)=1
$$

It is easy to check that then

$$
\gamma_{i, i+1}(t)+\gamma_{i+1, i+2}(t)=\gamma_{i, i+2}(t)
$$

where the orientation of $\gamma_{i, i+2}(t)$ is appropriately chosen. Therefore the orientations of the remaining cycles can be chosen to satisfy

$$
\gamma_{i j} \circ \gamma_{j k}=+1, \gamma_{i j}+\gamma_{j k}=\gamma_{i k}, \quad \forall i<j<k .
$$

As a by product we have also

$$
\gamma_{1,2}+\gamma_{2,3}+\cdots+\gamma_{n, 1}=0
$$

Obviously this fixes the orientation of all cycles $\gamma_{i j}$ up to simultaneous multiplication by -1 , which have no incidence on the result claimed in Corollary 4.2. The standard basis of vanishing cycles of the the singularity $y^{2}+x^{5}$ is shown on fig. 7 . We shall construct an isomorphism

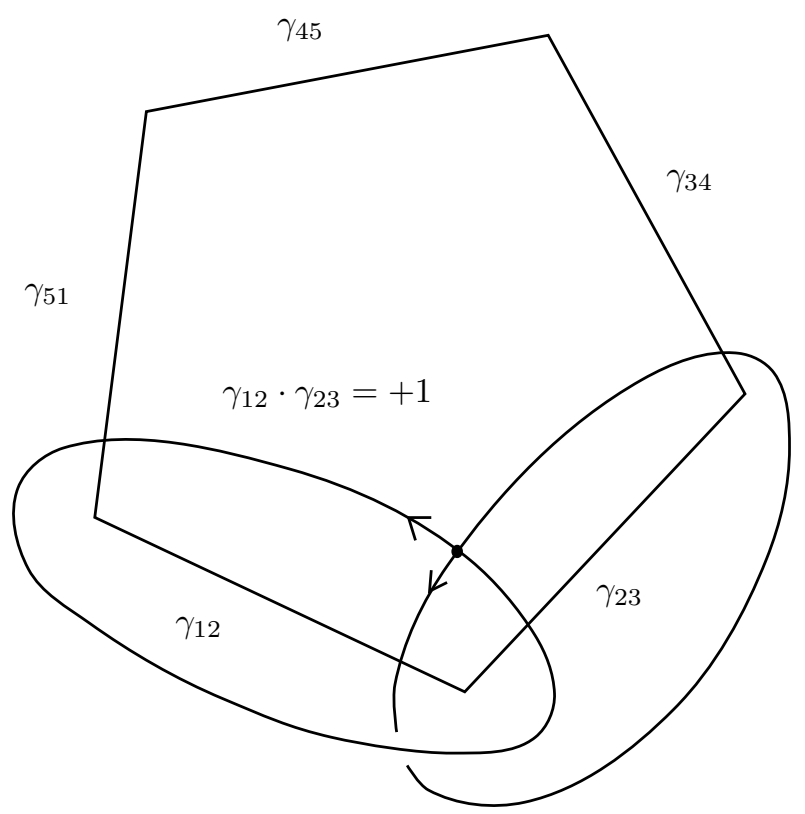

FIGURE 7 . The standard basis of vanishing cycles of the the singularity $y^{2}+x^{5}$.

$$
\begin{aligned}
H_{1}\left(\Gamma_{t}, \mathbb{Z}\right) & \rightarrow \widetilde{H}_{0}\left(f^{-1}(z), \mathbb{Z}\right) \\
\gamma(t) & \mapsto \delta(z)
\end{aligned}
$$


having the property announced in Corollary 4.2. According to the proof of Theorem 4.2 this should be a linear map which associates to the one-cycle $\gamma_{i j}(t)$ the reduced 0 -cycle (see also section 2) $\delta_{i j}(t)= \pm\left(f_{i}^{-1}(t)-f_{j}^{-1}(t)\right)$ and should be therefore compatible to the relations

$$
\gamma_{i j}+\gamma_{j k}=\gamma_{i k}, \quad \delta_{i j}+\delta_{j k}=\delta_{i k} .
$$

It follows that the orientation of the 0 -cycles $\delta_{i j}(t)$ can be fixed as

$$
\delta_{i j}(t)=f_{i}^{-1}(t)-f_{j}^{-1}(t), \quad \forall i<j .
$$

Note that the above isomorphism is not compatible to the monodromy action.

In conclusion, if

$$
\gamma(t)=\sum n_{i j} \gamma_{i j}(t)
$$

and $I(t)=\int_{\gamma(t)} \omega \equiv 0$ then $\int_{\delta(z)} K \equiv 0$ where

$$
\delta(z)=\sum n_{i j}\left(f_{i}^{-1}(z)-f_{j}^{-1}(z)\right) .
$$

4.3. Hyperelliptic Abelian integrals along ovals. Let $f(x) \in \mathbb{R}[x]$ be an arbitrary non-linear real polynomial. Consider a family of ovals $\{\gamma(t)\}_{t}$

$$
\gamma(t) \subset\left\{(x, y) \in \mathbb{R}^{2}: y^{2}-f(x)=t\right\}, t \in \mathbb{R}
$$

depending continuously on the real parameter $t$. Each oval $\gamma(t)$ can be parameterized as

$$
y= \pm \sqrt{f(x)+t}, \quad x_{1}(t) \leq x \leq x_{2}(t)
$$

where $x_{1}(t)<x_{2}(t)$ are two real roots of $f(x)+t$. The purpose of this last section is to solve, by making use of Theorem 4.1 and Theorem 4.2, the following problem: under what conditions the Abelian integral (45)

$$
I(t)=\int_{\gamma(t)} k(x) y d x=2 \int_{x_{1}(t)}^{x_{2}(t)} k(x) y d x
$$

is identically zero?

Theorem 4.3. The integral $I(t)$ vanishes identically if and only if there exists a polynomial $r \in \mathbb{R}[x]$, such that both $f$ and $K=\int k$ are polynomials in $r$, and $r\left(x_{1}(t)\right) \equiv r\left(x_{2}(t)\right)$.

Proof. First of all, note that if $K$ and $f$ have a right compositional factor identifying $x_{1}(t)$ and $x_{2}(t)$, then the Abelian integral $\int_{\gamma(t)} k(x) y d x$ is a pull back of an integral along a cycle homologous to zero, and hence vanishes identically.

Suppose further that $I(t)$ vanishes identically. It is enough to show that this implies $K\left(x_{1}(t)\right) \equiv K\left(x_{2}(t)\right)$ since in this case by Proposition 2.1 (or by the Lüroth theorem) $f$ and $K$ will have a right compositional factor identifying $x_{1}(t)$ and $x_{2}(t)$. If there exists a path on the complex $t$-plane along which the cycle $\gamma(t)$ vanishes, then Theorem 4.2 applies and we conclude that $K\left(x_{1}(t)\right) \equiv K\left(x_{2}(t)\right)$.

As an example, consider a real polynomial $f$ of degree $n \geq 2 k, f=-x^{2 k}+\ldots$. Let $x_{1}(t)<x_{2}(t)$ be the two real roots of $f(x)+t$ which tend to 0 as $t$ tends to zero and $\{\gamma(t)\}$ be the continuous family of ovals vanishing at the origin as $t$ tends to zero.

$$
\gamma(t) \subset\left\{(x, y) \in \mathbb{R}^{2}: y^{2}+x^{2 k}+\cdots=t\right\}
$$


Then Theorem 4.2 applies and hence the result of Theorem 4.3 follows. In the Morse case $(k=1)$, this has been proved by Christopher and Mardesic [3].

The condition that $\gamma(t)$ vanishes along a suitable path is essential, and holds for arbitrary real polynomials of degree four or five, see for instance [4, section 3.1 , where the case $f(x)=\left(x^{2}-1\right)^{2}$ is studied. We do not know whether this condition is fulfilled for arbitrary polynomial $f$ and family of ovals $\gamma(t)$. See Fig. 8 (continuous families of ovals). However, using Theorem 4.1 instead of Theorem 4.2 we can prove the theorem in its full generality.

Indeed, let $f$ be an arbitrary real polynomial of degree $n>1$ and $I(t)$ be an identically vanishing Abelian integral as before. Let us apply Theorem 4.1. For this purpose, let us fix a regular real value $t$ of $f$, and consider the moment problem associated to the oval $\gamma(t)$ on the Riemann surface $\Gamma_{t}$. Following the method described in section 2 we have to consider a constellation $\lambda_{f} \subset \mathbb{P}^{1}$ and to deform the image of $\gamma(t)$ under $f+t$ on $\lambda_{f}$. The closed loop $\gamma(t)$ being an oval, its image is just a real interval connecting 0 to a critical value $c_{k}$ of $f+t$. Suppose for instance that $0>c_{1}>c_{2}>\cdots>c_{k}$ are the remaining critical values of $f+t$ contained in $\left[c_{k}, 0\right]$. We have therefore

$$
\left[c_{k}, 0\right]=\left[c_{k}, c_{k-1}\right] \cup \cdots \cup\left[c_{1}, 0\right]
$$

which, without loss of generality, will be used on the place of the constellation $\lambda_{f}$. To each segment $\left[c_{i-1}, c_{i}\right]$ we associate a 0 -cycle $\delta_{i}$ and $I(t)$ is a rational function if and only if $\int_{\delta_{i}(z)} K \equiv 0$.

Example. The critical values of the polynomial $\left(x^{2} / 2-1\right)^{2}-1$ are -1 and $-3 / 4$. The relevant constellation associated to the exterior family of ovals shown on fig. 8 is $[-1,-3 / 4] \cup[-3 / 4,0]$. To the segment $[-3 / 4,0]$ we associate the 0 -cycle $\delta_{1}(z)=x_{1}(z)-x_{2}(z)$ and to the segment $[-1,-3 / 4]$ the 0 -cycle $\delta_{2}(z)=x_{1}(z)-x_{3}(z)+$ $x_{4}(z)-x_{2}(z)$.
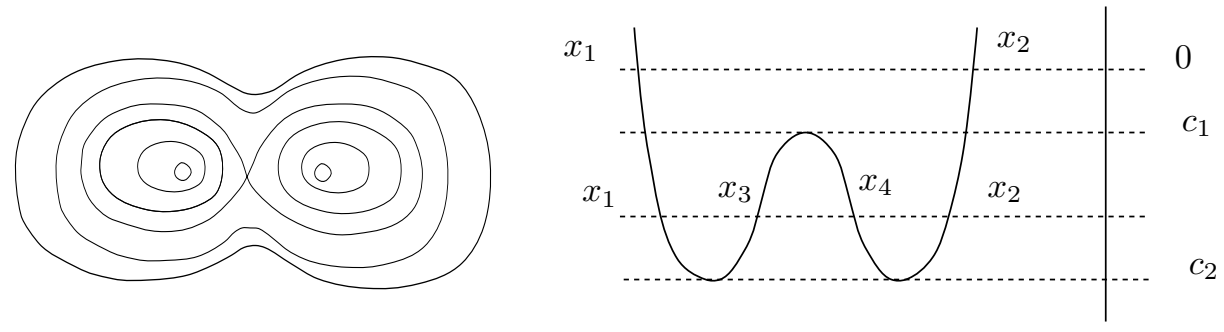

Figure 8. The continuous families of ovals of $y^{2}+\left(x^{2} / 2-1\right)^{2}$ and the graph of $\left(x^{2} / 2-1\right)^{2}-1$.

Fortunately in general we do not need to compute all of $\delta_{i}$. We note that the image of $\left[x_{1}(t), x_{2}(t)\right]$ is a closed curve covering $\left[c_{k}, 0\right]$. The pre-image of each point $z \in\left(0, c_{1}\right)$ consists of two points $x_{1}(z)$ and $x_{2}(z)$ (roots of $\left.f(x)+t-z\right)$ and hence $\delta_{1}(z)=x_{1}(z)-x_{2}(z)$. We conclude that $K\left(x_{1}(t)\right) \equiv K\left(x_{2}(t)\right)$ which completes the proof of Theorem4.3. 


\section{REFERENCES}

1. A. Alvarez, J. Bravo, P. Mardesic, Persistent and tangential center problem and abelian integrals in dimension zero, arXiv:1101.1777.

2. M. Briskin, N. Roytvarf, and Y. Yomdin, Center conditions at infinity for Abel differential equations, Ann. of Math. (2) $\mathbf{1 7 2}$ (2010), no. 1, 437-483.

3. C. Christopher, P. Mardeshich, The monodromy problem and the tangential focus problem, Funktsional. Anal. i Prilozhen. 44 (2010), no. 1, 27-43.

4. L. Gavrilov and I.D. Iliev, The displacement map associated to polynomial unfoldings of planar Hamiltonian vector fields, American J. of Math., 127 (2005) 1153-1190.

5. L. Gavrilov, Higher order Poincaré-Pontryagin functions and iterated path integrals, Ann. Fac. Sci. Toulouse Math. (6) 14 (2005), no. 4, 663-682.

6. L. Gavrilov and H. Movasati, The infinitesimal 16th Hilbert problem in dimension zero, Bull. Sci. Math. 131 (2007), no. 3, 242-257.

7. L. Gavrilov, H. Movasati, and I. Nakai, On the non-persistence of Hamiltonian identity cycles, J. Differential Equations 246 (2009), no. 7, 2706-2723.

8. S. Lando, A. Zvonkin, Graphs on surfaces and their applications, Encyclopaedia of Mathematical Sciences, 141. Low-Dimensional Topology, II. Springer-Verlag, Berlin, 2004.

9. H. Movasati, Center conditions: rigidity of logarithmic differential equations, J. Differential Equations 197 (2004), no. 1, 197-217.

10. F. Pakovich, On the polynomial moment problem, Math. Research Letters 10, (2003), 401-410.

11. F. Pakovich, N. Roytvarf, and Y. Yomdin, Cauchy-type integrals of algebraic functions, Israel J. Math. 144 (2004), 221-291.

12. F. Pakovich, On polynomials orthogonal to all powers of a Chebyshev polynomial on a segment, Israel J. Math., Vol. 142 (2004), 273-283

13. F. Pakovich, On polynomials orthogonal to all powers of a given polynomial on a segment, Bull. Sci. Math. 129 (2005), 749-774.

14. F. Pakovich, M. Muzychuk, Solution of the polynomial moment problem, Proc. Lond. Math. Soc., V. 99 no. 3, 633-657 (2009).

15. F. Pakovich, On rational functions orthogonal to all powers of a given rational function on a curve, preprint,arXiv:0910.2105.

16. N. Roytvarf, Generalized moments, composition of polynomials and Bernstein classes, Entire functions in modern analysis (Tel-Aviv, 1997), 339355, Israel Math. Conf. Proc., 15, Bar-Ilan Univ., Ramat Gan, 2001.

Institut de Mathématiques de Toulouse, UMR 5219, Université de Toulouse, 31062 Toulouse, France

E-mail address: lubomir.gavrilov@math.univ-toulouse.fr

Department of Mathematics, Ben-Gurion University of the Negev, P.O.B. 653, BeerSheVA, IsRAel; E-MAIL:

E-mail address: pakovich@math.bgu.ac.il 